\title{
CHEBYSHEV TYPE INEQUALITY ON NABLA DISCRETE FRACTIONAL CALCULUS
}

\section{Serkan Asliyüce And Ayşe FeZA Güvenilir}

Abstract. In this paper, we establish some Chebyshev type inequalities on discrete fractional calculus with nabla operator (or backward difference operator).

Mathematics subject classification (2010): Primary 26D15, 26A33; Secondary 39A12, 26D10. Keywords and phrases: Chebyshev inequality, nabla operator, discrete fractional calculus.

\section{REFERENCES}

[1] E. Akin, S. Asliyüce, A. F. GÜVenİlİR, B. KaymakÇalan, Discrete Grüss type inequality on fractional calculus, J. Ineq. Appl. 174, (2015), 7 pp.

[2] G. A. Anastassiou, Nabla discrete fractional calculus and nabla inequalities, Math. Comput. Modelling 51, (2010), 562-571.

[3] F. M. Atici P. W. Eloe, A transform method in discrete fractional calculus, Int. J. Difference Equ. 2, (2007), 165-176.

[4] F. M. Atici P. W. Eloe, Initial value problems in discrete fractional calculus, Proc. Amer. Math. Soc. 137, (2009), 981-989.

[5] F. M. Atici P. W. Eloe, Discrete fractional calculus with the nabla operator, Electron J. Qual. Theory Differ. Equ. 3, (2009), 12pp.

[6] F. M. Atici S. ŞEngüL, Modeling with fractional difference equations, J. Math. Anal. Appl. 369, (2010), 157-159.

[7] F. M. Atici P. W. Eloe, Gronwall's inequality on discrete fractional calculus, Comput. Math. Appl. 64, (2012), 3193-3200.

[8] N. R. O. Bastos, R. A. C. Ferreira, D. F. M. Torres, Necessary optimality conditions for fractional difference problems of the calculus of variations, Discrete Contin. Dyn. Syst. 29, (2011), 417-437.

[9] P. L. Chebyshev, Sur les expressions approximatives des integrales definies par les autres prises entre les memes limites, Proc. Math. Soc. Charkov 2, (1882), 93-98.

[10] G. V. S. R. Deekshitulu, J. J. Mohan, Fractional difference inequalities of Bihari type, Commun. Appl. Anal. 14, (2010), 343-354.

[11] J. B. DiaZ, T. J. OsLER, Differences of fractional order, Math. Comp. 28, (1974), 185-202.

[12] Q. FENG, Some new generalized Gronwall-Bellman type discrete fractional inequalities, Appl. Math. Comput. 259, (2015), 403-411.

[13] R. A. C. Ferreira, A discrete fractional Gronwall inequality, Proc. Amer. Math. Soc. 140, (2012), $1605-1612$.

[14] C. S. Goodrich, Continuity of solutions to discrete fractional initial value problems, Comput. Math. Appl. 59, (2010), 3489-3499.

[15] H. L. Gray, N. F. ZhANG, On a new definition of fractional difference, Math. Comp. 50, (1988), $513-529$.

[16] A. F. GÜvenİlİR, B. Kaymakçalan, A. C. Peterson, K. Taş, Nabla discrete fractional Grüss type inequality, J. Ineq. Appl. 86, (2014), 9 pp.

[17] M. Holm, Sum and difference compositions in discrete fractional calculus, Cubo 13, (2011), 153184. 